\title{
Analgesic abuse headache: risk factors and causes of poor adherence in the detox process
}

\author{
Klairton Duarte de Freitas (D), Raimundo Neudson Maia Alcantara (D) \\ Hospital Geral de Fortaleza, Ceará, Brazil
}

\section{$凶$}

Klairton Duarte de Freitas

Departamento de Neurologia, Hospital Geral de Fortaleza Rua Ávila Goulart, 900, Papicu, Fortaleza, CE, Brazil CEP: $60175-295$

Phone +55 85 99690-1485

klairton-duarte@hotamil.com

Edited by:

Marcelo Moraes Valença

Keywords:

Medication-overuse headache

Risk factors

Comorbidities

Poor adherence

Dependence

Headache Disorders

\begin{abstract}
Background

Medication overuse headache $(\mathrm{MOH})$ is characterized by a pre-existing primary or secondary headache associated with medication overuse.

Aims

To identify the clinical, epidemiological, and therapeutic profiles associated with $\mathrm{MOH}$ and poor adherence to treatment.

Methods

A cross-sectional, comparative, descriptive, analytical study was carried out to assess the characteristics of patients with $\mathrm{MOH}$ treated at the Hospital Geral de Fortaleza (HGF). Results

103 patients participated, 95 (92.2\%) women and 8 (7.8\%) men. Of these, 55 (53.4\%) patients answered that had already been instructed about the $\mathrm{MOH}$, however they continue to abuse medication for many reasons, i.e.: difficulty in bearing pain, and fear of worsening the pain. When asked about what could be done to improve adherence to treatment, in a general way, $28(27.2 \%)$ were unable to inform, $37(35.9 \%)$ answered that most frequent consultations could help. Furthermore, 19 (18.4\%) believe that psychological counseling wound bring benefits.

\section{Conclusion}

The lack of guidance or interest in the guidelines provided are real and important obstacles to treat $\mathrm{MOH}$. Changes in the care model that include effective communication, more frequent return, family and psychotherapy support and close monitoring by the physician or nurses are factors that should be considered in headache clinics.
\end{abstract}




\section{Introduction}

Leadaches are a major public health concern given their high prevalence ( $46 \%$ of the population), usually occurring episodically, but $2 \%$ to $5 \%$ of the population have chronic headache. In these patients the excessive or prolonged use of drugs for symptomatic treatment of headaches can be the cause of headache by abuse of painkillers. ${ }^{1,2}$

Medication overuse headache $(\mathrm{MOH})$ is characterized in a patient with pre-existing primary or secondary headache, in which, associated with the excessive use of medication, a new type of headache develops, or pre-existing headache worsens, the following criteria being present: a) headache present for more than 15 days per month; b) regular overuse of analgesics for more than 3 months, c) headache is not better explained by another diagnosis and developed or worsened during the excessive use of medication. Ingestion of 15 days or more per month of simple analgesics and combination acute medications is considered for a diagnosis of $\mathrm{MOH}$, and for triptans, ergotamine, opioids, and combination analgesics, 10 days per month is sufficient. ${ }^{3}$

Prevalence rates of $\mathrm{MOH}$ in the European population are between $1-2 \%$, but in specialized headache centers, they range from $30 \%$ to $70 \%$, a high percentage of abuse, even for specialized centers. ${ }^{4 \cdot 6}$ An important factor to consider in the abuse of analgesics is pain prevention. For example, if there is medical advice for early use at the onset of pain, the patient may understand that there is freedom to use without quantity limitation or understand that there is a justification for use. In this sense, the study of Sousa ${ }^{7}$ cites some reasons for abuse, such as difficulty enduring pain, fear of arising pain if you do not take the medication, reappearance of pain, not believing in the cure, better performance at work, help with sleep, and decrease anxiety. ${ }^{7}$

However, regardless of individual reasons for symptomatic drug overuse, there are important risk factors that have been proposed for the development of $\mathrm{MOH}$. For example, the ratio between men and women is $1: 3-4^{8}$ and the condition is most prevalent in the fourth decade of life; however it tends to decrease with age, reaching prevalence between $1.0 \%$ and $1.7 \%$ in those over 65 years of age. ${ }^{4,5,8} \mathrm{We}$ can also cite as associated factors low educational level, unemployment, marital status, high levels of daily stress, low socioeconomic level, alcoholism, daily smoking, family history of $\mathrm{MOH}$ or abuse of other substances. ${ }^{9}$

Among the primary headaches, migraine was responsible for $65 \%$ of the $\mathrm{MOH}$ cases. Regarding the type of analgesics, it was observed that the use of triptans was more associated with the development of $\mathrm{MOH}$ compared to simple analgesics. Regarding clinical complaints, cardiovascular risk factors, as well as respiratory diseases, gastrointestinal complaints, insomnia, chronic musculoskeletal pain, and other bodily pain, increase the risk of abuse. Finally, psychiatric comorbidities and genetic risk factors, such as homozygous D/D ACE (angiotensinconverting enzyme) polymorphism, which correlated positively with $\mathrm{MOH}$ duration and Val66Met BDNF polymorphism which predicted consumption of painkiller use, are also causes of $\mathrm{MOH} .{ }^{10-12}$

Given the above, we can see that many psychosocial and socioeconomic factors are associated with $\mathrm{MOH}$. However, it is difficult to determine whether these are directly or indirectly associated, since these findings are mainly based on cross-sectional studies. Thus, most of these factors may merely be markers of a complex situation, since many aspects of life may be affected by chronic headache as with other conditions. ${ }^{13}$

In this sense, it is important to note that despite the existence of multiple risk factors associated with $\mathrm{MOH}$, ignorance about the harmful effects of overuse of analgesics, even for other clinical conditions, has great potential to cause $\mathrm{MOH}$. On the other hand, why do people who have already been educated about the risk of analgesic abuse continue to do so? Thus, the objective of this study is to identify the clinical, epidemiological, and therapeutic profile related to $\mathrm{MOH}$ and the poor adherence to its treatment.

\section{Methods}

\section{Research Design}

This is a cross-sectional, comparative, descriptive analytical study that evaluated the characteristics of the population of patients with $\mathrm{MOH}$, registered and regularly accompanied in the Headache Outpatient Clinic of the HGF, in the city of Fortaleza, Ceará, Brazil. A questionnaire was applied during outpatient consultations and by telemedicine during the period from March to May 2021.

\section{Research Population}

The study population was composed of patients regularly enrolled and accompanied at the headache outpatient 
clinic of the HGF. The inclusion criteria were: $\mathrm{MOH}$ confirmed by the ICHD-3 criteria, patients over 18 years of age, of both sexes, registered at the mentioned center. The study was approved by the Ethics Committee of the HGF (4.568.755). Due to the pandemic of COVID-19, the application of the Informed Consent Form (ICF), occurred through Google forms to facilitate the start of data collection.

\section{Statistical Analysis}

Numerical variables were described by measures of central tendency and variability. Categorical variables were represented by frequency distribution. All statistical analyses were performed using Microsoft Excel version 2010 software.

\section{Results}

The study population consisted of $95(92.2 \%)$ women and $8(7.8 \%)$ men, for a total of 103 patients. The patients were grouped by age intervals, with the groups aged 40 49 years and 50-59 years having the most participants, totaling 55 patients (53.4\%). Of the 103 patients, 57 (55.3\%) had less than complete high school education, $65(63.1 \%)$ were married or in a stable union, and 85 (82.5\%) were not employed during the study. The main type of primary headache was migraine (93 patients, $90.3 \%$ ), with onset more than 10 years ago (68 patients, $66 \%$ ) and headache frequency more than five days a week (47 patients, 45.6\%). For headache control, 47 (46.5\%) patients used common analgesics and $83(80.6 \%)$ did not use analgesics for any condition other than headache (Tables 1 and 2).

More than half of the patients who took part in the study (55 patients, 53.4\%), answered that they had already been oriented about the excessive use of drugs and even so, they continued to abuse them for various reasons, among which the difficulty in bearing the pain and fear of worsening their headaches were the most cited. Regarding therapy for detoxification, of the 103 participants, 67 $(65 \%)$ did not do it, 5 (4.9\%) did not remember, and 31 (30.1\%) answered that they had gone through at least one therapy during their follow-up. When asked about prophylactic medication, $69(67 \%)$ said they have used it, with 33 patients citing tricyclic antidepressants and 41 patients citing anti-crisis drugs as the most used, remembering that the same patient could take more than one class of drugs. Thus, among those who did not take prophylactic medication, most of the answers mentioned side effects (11 answers) and the belief that the medication
Table 1. Sociodemographic variables related to medication overuse headache $(\mathrm{MOH})$

\begin{tabular}{|c|c|c|c|}
\hline \multicolumn{2}{|r|}{ Variables } & $\mathbf{n}$ & $\%$ \\
\hline \multirow{2}{*}{ Sex } & Male & 8 & 7.8 \\
\hline & Female & 95 & 92.2 \\
\hline \multirow{5}{*}{ Age (years) } & $18-30$ & 12 & 11.7 \\
\hline & $31-39$ & 20 & 19.4 \\
\hline & $40-49$ & 28 & 27.2 \\
\hline & $50-59$ & 27 & 26.2 \\
\hline & $\geq 60$ & 16 & 15.5 \\
\hline \multirow{7}{*}{ Level of Education } & Illiterate & 3 & 2.9 \\
\hline & Elementary School (incomplete) & 34 & 33.0 \\
\hline & Elementary School (complete) & 11 & 10.7 \\
\hline & High School (incomplete) & 9 & 8.7 \\
\hline & High School (complete) & 32 & 31.1 \\
\hline & Superior (incomplete) & 3 & 2.9 \\
\hline & University (complete) & 11 & 10.7 \\
\hline \multirow{3}{*}{ Marital Status } & Married & 65 & 63.1 \\
\hline & Single & 26 & 25.2 \\
\hline & Divorced or Widowed & 12 & 11.7 \\
\hline \multirow{2}{*}{ Employee } & Yes & 18 & 17.5 \\
\hline & No & 85 & 82.5 \\
\hline
\end{tabular}

Table 2. Variables related to primary headache and analgesics used for medication overuse headache $(\mathrm{MOH})$

\begin{tabular}{|c|c|c|c|}
\hline \multicolumn{2}{|c|}{ Variables } & $\mathbf{n}$ & $\%$ \\
\hline \multirow{4}{*}{$\begin{array}{l}\text { Type of primary } \\
\text { headache }\end{array}$} & Migraine & 93 & 90.3 \\
\hline & Tension-type headache & 0 & 0 \\
\hline & Cluster headache & 1 & 1.0 \\
\hline & Others & 9 & 8.7 \\
\hline \multirow{3}{*}{$\begin{array}{c}\text { Duration of } \\
\text { headache (years) }\end{array}$} & $<2$ & 6 & 5.8 \\
\hline & $2-10$ & 29 & 28.2 \\
\hline & $>10$ & 68 & 66.0 \\
\hline \multirow{3}{*}{$\begin{array}{c}\text { Frequency of } \\
\text { headache (days/ } \\
\text { week) }\end{array}$} & $<3$ & 18 & 17.5 \\
\hline & $4-5$ & 38 & 36.4 \\
\hline & $>5$ & 47 & 45.6 \\
\hline \multirow{6}{*}{$\begin{array}{c}\text { Type of } \\
\text { analgesics }\end{array}$} & Simple & 47 & 45.6 \\
\hline & Combined & 23 & 22.3 \\
\hline & Multiples & 32 & 31.1 \\
\hline & Triptans & 1 & 1.0 \\
\hline & Opioids & 0 & 0 \\
\hline & Ergotamines & 0 & 0 \\
\hline \multirow{3}{*}{$\begin{array}{l}\text { Analgesics } \\
\text { used for other } \\
\text { conditions }\end{array}$} & Not use & 83 & 80.6 \\
\hline & MS* pains & 14 & 13.6 \\
\hline & Others & 6 & 5.8 \\
\hline
\end{tabular}

*musculoskeletal 
would have no effect (10 answers). As for habits, there was no report of illegal drug use, but smoking, drinking alcohol, and drinking coffee more than 3 times a day were mentioned. In addition, most of the answers associated $\mathrm{MOH}$ with insomnia, a sedentary lifestyle, obesity, and a family history of headaches, among others. Regarding psychiatric diagnoses (based on DSM-V), 8 (7.8\%) patients were diagnosed with depression, $26(25.2 \%)$ with anxiety, and $38(36.9 \%)$ with depression and anxiety. On the other hand, 31 (30.1\%) did not present depression and/or anxiety. In the same way, from 103 patients, 75 (72.8\%) presented some clinical comorbidity, being cardiovascular, respiratory, and musculoskeletal diseases the most reported (Table 3) (Figures 1, 2, 3 and 4).

Of the 103 patients, 37 (35.9\%) had follow-ups between 6-12 months, 27 (26.2\%) had follow-ups between 3-6 months. Only $21(20.4 \%)$ attended the headache outpatient clinic for less than 3 months. About the follow-up in primary care, $66(64.1 \%)$ patients did not follow up and when asked what could be done to improve adherence to detoxification treatment, 28 (27.2\%) did not know, while $37(35.9 \%)$ and 19 (18.4\%) answered, respectively, that more frequent consultations and psychological follow-up could help (Table 3).

Table 3. Clinical data from the anamnesis

\begin{tabular}{|c|c|c|c|}
\hline \multicolumn{2}{|l|}{ Variables } & \multirow{2}{*}{$\frac{\mathbf{n}}{55}$} & \multirow{2}{*}{$\begin{array}{c}\% \\
53.4\end{array}$} \\
\hline Have you ever been quided about the & Yes & & \\
\hline abusive use of painkillers? & No & 48 & 46.6 \\
\hline \multirow{3}{*}{ Have you ever had detox therapy? } & Yes & 31 & 30.1 \\
\hline & No & 67 & 65.0 \\
\hline & Do not remember & 5 & 4.9 \\
\hline \multirow{2}{*}{ Do you use prophylactic medication? } & Yes & 69 & 67.0 \\
\hline & No & 34 & 33.0 \\
\hline \multirow{4}{*}{ Psychiatric comorbidities } & No & 31 & 30.1 \\
\hline & Depression & 8 & 7.8 \\
\hline & Anxiety & 26 & 25.2 \\
\hline & $\mathrm{DA}^{*}$ & 38 & 36.9 \\
\hline \multirow{2}{*}{ Clinical comorbidities } & Yes & 75 & 72.8 \\
\hline & No & 28 & 27.2 \\
\hline \multirow{4}{*}{ Outpatient follow-up (months) } & $<3$ & 21 & 20.4 \\
\hline & $3-6$ & 27 & 26.2 \\
\hline & $6-12$ & 37 & 35.9 \\
\hline & $>12$ & 18 & 17.5 \\
\hline \multirow{5}{*}{ Follow-up in primary care } & $<3$ & 22 & 21.4 \\
\hline & $3-6$ & 7 & 6.8 \\
\hline & $6-12$ & 6 & 5.8 \\
\hline & $>12$ & 2 & 1.9 \\
\hline & No & 66 & 64.1 \\
\hline
\end{tabular}

*Depression and anxiety

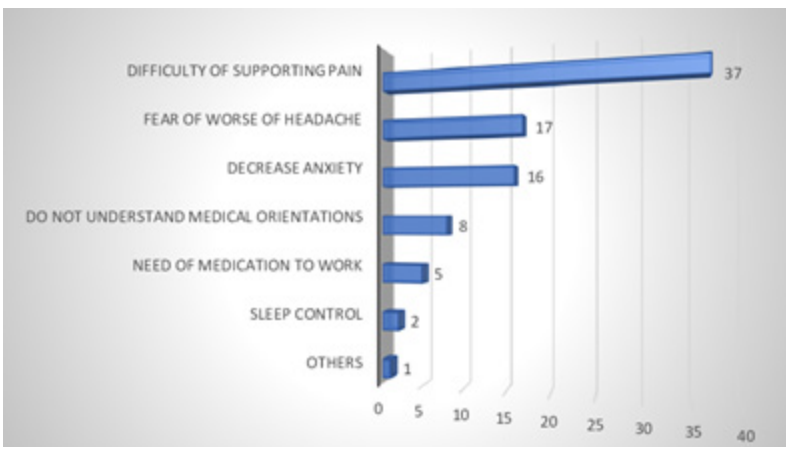

Figure 1. Cause of the permanent use of analgesics.

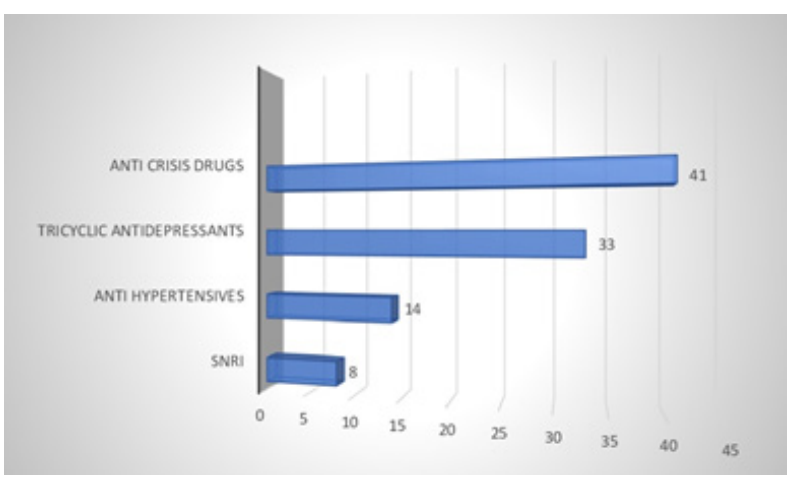

Figure 2. Prophylactic drugs.

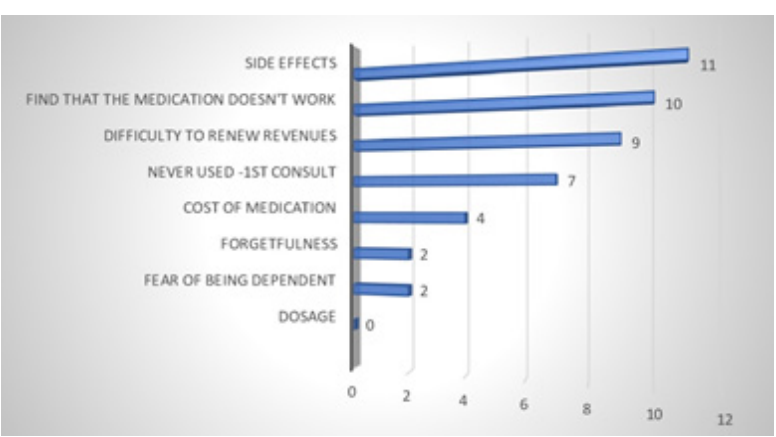

Figure 3. Causes of non-adherence to prophylactic medication

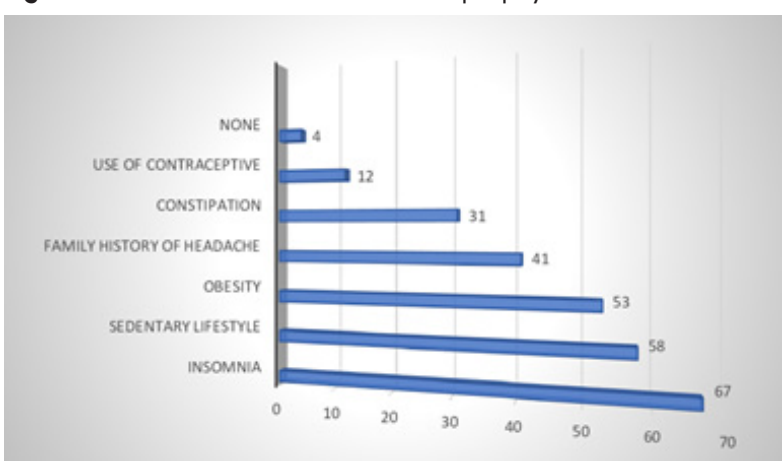

Figure 4. Risk factors associated with medication overuse headache 


\section{Discussion}

$\mathrm{MOH}$ is a chronic disorder that can impact the patient's quality of life both socially and financially. Prevalence rates are similar in most populations studied, with a preponderance of female sex, average age ranging between fourth and fifth decades of life, married, low educational level when compared to the general population, and most often without paid occupation. 8,9,14,15 Looking only at sex, the guideline of the European Academy of Neurology published a recent study where the majority of patients with $\mathrm{MOH}$ are female, 93\%. ${ }^{6}$ In the present study it was no different, most of the patients followed were female, $92.2 \%$, and belonging to the fourth and fifth decade of life, totaling 55 patients, 53.4\%. Of the 103 patients, 57 (55.3\%) had less than complete high school education, $65(63.1 \%)$ were married or in a stable union, and 85 $(82.5 \%)$ were not employed during the study.

Another important factor when it comes to risk factors is the type of primary headache, when it started and the frequency. A meta-analysis of 29 studies showed that migraine was the most common type of primary headache, around $65 \%$, followed by tension-type headache $27 \%{ }^{5}$ Further studies 6,16 find similar data, where $80 \%$ of the patients had migraine as their primary headache. To Nagen et al. ${ }^{15}$, migraine was also the most frequent primary headache. These data should be interpreted depending on where the studies were conducted. For example, our work, as well as the studies cited above, were performed in a tertiary hospital, and similar results were found here $(90.3 \%$ patients with migraine). On the other hand, tension-type headache, considered the most common primary headache, was not mentioned in any of our patients, and the other $9.7 \%$ was distributed between cluster headache, idiopathic intracranial hypertension, cervicogenic headache, and post-traumatic headache. In addition, as expected, long-standing and more frequent headache at the initial visit is associated with higher risk of developing $\mathrm{MOH}$ than infrequent headache. ${ }^{17}$ Our results showed that most patients, $66 \%$ had headache onset more than 10 years ago and $82.5 \%$ reported headache for 4 days or more per week, indicating a higher possibility of committing abuse.

Besides these risk factors, the easy access to acquire certain medications and to make combinations of them apply. In our study, common analgesics $(45.6 \%)$, followed by multiple analgesics $(31.1 \%)$ and then combined analgesics (22.3\%) were the most used. Despite being in a tertiary hospital, where the use of triptans, or opioids, would be more common, our patients have low purchasing power, which justifies the use of common analgesics as the main medication, a fact corroborated by comparative studies between Europe and Latin America, where 31\% versus $6 \%$ of patients abuse triptans. Also in our study group, only $1 \%$ of patients abused triptans alone and no patients abused ergotamine or barbiturates alone. However, for multiple analgesics, the association of common analgesics with triptans, opioids and ergotamine predominated. Thus, when comparing these data with data from other studies, cultural, economic, and political differences may contribute to international variation in medication use. In Spain, simple analgesics are most used, followed by ergotamine among patients with $\mathrm{MOH}$. Scandinavian studies show the same trend toward a high preference for simple analgesics, accompanied by combination analgesics. In the USA it has been described that patients with $\mathrm{MOH}$ use more opioids and barbiturates than in other nations. ${ }^{18}$ However, excessive consumption of analgesics, combined or not, may be associated with the presence of comorbidities.

Thus, in relation to self-reported comorbidities, 20 (19.4\%) patients took analgesics frequently (at least once a week) for conditions other than headache. Among them, 14 patients related taking them for musculoskeletal and connective tissue pain, 5 patients for non-neoplastic pain of abdomino-pelvic origin, and 1 patient was taking medication not to combat pain but to control chronic daily cough secondary to lung neoplasm. In our results, the major associated comorbidities were cardiovascular diseases (hypertension, dyslipidemia, diabetes, stroke), respiratory (asthma, allergic rhinitis, COPD), and musculoskeletal (herniated disc, osteoarthritis, fibromyalgia, and rheumatoid arthritis). Besides comorbidities, the risk and psychosocial factors most often mentioned in our study were: smoking, alcoholism, insomnia, obesity, sedentariness and family history of headache, a fact corroborated by other studies. ${ }^{10.12}$ And when looking at the amount of psychiatric disorders, $\mathrm{MOH}$ patients seem to be more likely to have multiple psychiatric comorbidities. ${ }^{5}$ In this context, our patients showed more pathological personality characteristics, according to the application of the DSM V diagnostic criteria for depression and anxiety. The observed prevalence of the evaluated psychiatric disorders was 72 (69.9\%) patients, of these 8 (7.8\%) patients had depression; 26 (25.2\%) anxiety; 38 (36.9\%) both disorders. While 31 (30.1\%) patients did not close criteria for depression nor anxiety but could not be ruled out from other unevaluated pathological personalities. Similar results were observed in other studies. 5,9,19 
But the biggest question is why the patient abuses medication. We know the risk factors, we know that comorbidities influence analgesic consumption, that psychosocial disorders make the patient more susceptible, and yet the rates seem to increase. It is necessary to understand if there is any factor related to the patient's knowledge of what abuse is, or if it is important to determine to what extent personality disorders are a consequence of inadequate treatment, or a cause of $\mathrm{MOH}$, and whether they should be more widely attributed to not only $\mathrm{MOH}$, but chronic pain in general. And, if there is knowledge about it, then it is important to understand why the patient continues to overuse it.

In this sense, in our study, $46.6 \%$ of the patients reported not knowing the effect of the abusive use of the medications. Other studies have presented similar results. In the study by Johnson et al. ${ }^{20}, 50 \%$ of patients who had seen a neurologist in the previous year denied having been instructed about the abuse of pain medications. In that same respect, Lai et al. ${ }^{21}$ observed that $78 \%$ of the respondents, whether headache sufferers or not, did not know the relationship between headache and analgesic abuse, and in the Bekkelund et al. ${ }^{22}$ only 2/262 (0.8\%) patients, after consultation with a neurologist, were informed by the specialist that they suffered from a possible headache associated with excessive use of analgesics. The authors suggest that the patients may have been informed but may not have remembered or fully understood the information. Thus, we wonder to what extent the patients were not informed, did not remember, did not understand the orientations, or due to their own abusive personality maintained the abuse. On the other hand, in some cases there is medical advice to start medication early before the crisis sets in. In our sample, 55 (53.4\%) patients continued to abuse medication, even under counseling. Among the causes for maintaining abuse, the most cited was difficulty in coping with pain (37 answers), followed by fear of worsening headache (17 answers), anxiety control (16 answers), not understanding guidelines (8 answers), and need to work ( 5 answers). Our results are similar to those shown by the review study by Sousa ${ }^{7}$, where the difficulty in bearing pain (67\%) is the most frequent answer, followed by the belief that there is no cure (65\%), the fear of feeling pain $(62 \%)$, the need for painkillers to work better $(62 \%)$, medical advice to take painkillers at the right time $(57 \%)$, to reduce anxiety (45\%), and the fear of worsening pain (30\%).

A fact that draws attention in our study is the number of patients who report not having received detoxification therapy (65\%) since therapy, either with a complete two-month cessation of acute medications or only with reduced medication intake, has been shown to be effective in reducing the frequency of headache days/month, converting a chronic migraine into an episodic one. Studies show a reduction in headache chronicity from $55 \%$ to $18 \%$ and in over $80 \%$ of cases patients were cured of $\mathrm{MOH}$ after 6 months. ${ }^{23}$

Medication adherence is essential to successful treatment of $\mathrm{MOH}$; analyses indicate that only $56 \%$ of patients adhere to treatment over 6 to 12 months with lower rates over time. In our survey, $33 \%$ of patients were not currently on any prophylactic medication. The fact is that the absence of therapy makes it difficult for the patient to adhere, especially in the first 10 days of detoxification due to withdrawal effects.

Another important point to be questioned would be regarding follow-up. In our study, we observed that most patients $(n=66,64.1 \%)$ had no follow-up in primary care, and only $22(21.4 \%)$ had a follow-up of less than 3 months. In our view, the lack of return to the consultations can become a risk factor, because without the ideal followup, the patient cannot renew the prescription, has no control of side effects or dose adjustment, nor support in moments of weakness. When we evaluated at the tertiary level, 55 (53.4\%) patients returned in a period longer than 6 months. That is, regardless of the level of care, the patients did not follow up optimally.

\section{Conclusion}

In our study, we observed that patients continue to abuse pain medication regardless of where they are seen and whether the physician is a generalist or a specialist, despite a clear lack of support from the patient's primary care. When asked what could be done to improve their adherence to treatment, the majority answered frequent return visits and psychological follow-up. In addition, we associated the lack of orientation or the failure to understand or interest in the orientations given, and the irregular use of medications as real and important obstacles.

In this sense, some studies have shown that patients submitted to drug therapy associated with non-pharmacological treatments, prevented relapses after 8 weeks of followup, when compared to drug therapy alone. ${ }^{24}$ Among the studies, we highlight those in which the patients received dynamic psychotherapy, frequent support (possibility of contacting the physician and/or team nurse), and the use of an electronic diary, focusing on patient education. Thus, 
the patient becomes more active in the decisions of when and how to treat and also collaborates in behavioral and lifestyle changes. ${ }^{25}$ Comparing these data with our results, we suggest changing the model of care by adopting measures that intensify and improve treatment adherence. These measures include effective communication, such as asking the patient to repeat important information, reinforcing concepts and key words, and making sure that the patient has understood the main directions. If necessary, making use of supplementary written material, more frequent feedback, family and psychotherapy support, and close follow up with the physician or nurse practitioner if the feedback is longer.

However, despite similar outcome indicators, the differences between the populations studied should be considered. For this, more studies are needed, with a longer follow-up time, for safer answers that can bring real benefit.

Klairton Duarte de Freitas

https://orcid.org/0000-0002-2143-1278

Raimundo Neudson Maia Alcantara

https://orcid.org/0000-0003-1927-926X

\section{References}

1. Kristoffersen ES and Lundqvist $C$. Medication-overuse headache: a review. J Pain Res 2014;7:367-378 Doi:10.2147/ipr.S46071

2. Stovner L, Hagen K, Jensen R, Katsarava Z, Lipton R, Scher $A, \ldots$ Zwart JA. The global burden of headache: a documentation of headache prevalence and disability worldwide. Cephalalgia 2007;27(3):193210 Doi:10.1111/j.1468-2982.2007.01288.x

3. Headache Classification Committee of the International Headache Society (IHS). The International Classification of Headache Disorders, 3rd edition. Cephalalgia 2018;38(1):1-211 Doi: $10.1177 / 0333102417738202$

4. Grazzi L, Andrasik F, D'Amico D, Leone $M$, Usai S, Kass SJ and Bussone G. Behavioral and pharmacologic treatment of transformed migraine with analgesic overuse: outcome at $\mathbf{3}$ years. Headache 2002;42(6):483-490 Doi:10.1046/ j. 1526-4610.2002.02123.x

5. Vandenbussche N, Laterza D, Lisicki M, Lloyd J, Lupi C, Tischler H, . . . Katsarava Z. Medicationoveruse headache: a widely recognized entity amidst ongoing debate. J Headache Pain 2018;19(1):50 Doi:10.1186/s10194-018-0875-x

6. Diener HC, Antonaci F, Braschinsky M, Evers S,
Jensen $R$, Lainez $M$, . . Petersen JA. European Academy of Neurology guideline on the management of medication-overuse headache. Eur I Neurol 2020;27(7): 11 102-1 116 Doi:10.1111/ene. 14268

7. Sousa LD. Cefaleias por abuso medicamentoso: aspectos clínicos. Sinapse 2002;2(1):1-5

8. Straube A, Pfaffenrath V, Ladwig KH, Meisinger C, Hoffmann W, Fendrich K, . . Berger K. Prevalence of chronic migraine and medication overuse headache in Germany--the German DMKG headache study. Cephalalgia 2010;30(2):207-213 Doi:10.1111/ j. 1468-2982.2009.01906.x

9. Evers $S$ and Marziniak $M$. Clinical features, pathophysiology, and treatment of medicationoveruse headache. Lancet Neurol 2010;9(4):391401 Doi:10.1016/s1474-4422(10)70008-9

10. Shand B, Goicochea MT, Valenzuela R, Fadic R, Jensen R, Tassorelli $C$ and Nappi $G$. Clinical and Demographical Characteristics of Patients with Medication Overuse Headache in Argentina and Chile: Analysis of the Latin American Section of COMOESTAS Project. J Headache Pain 2015; 16:83 Doi:10.1186/s10194-015-0561-1

11. Chen PK and Wang SJ. Medication Overuse and Medication Overuse Headache: Risk Factors, Comorbidities, Associated Burdens and Nonpharmacologic and Pharmacologic Treatment Approaches. Curr Pain Headache Rep 2019;23(8):60 Doi:10.1007/s $11916-019-0796-7$

12. Diener HC, Dodick D, Evers S, Holle D, Jensen RH, Lipton RB, ... Schwedt T. Pathophysiology, prevention, and treatment of medication overuse headache. Lancet Neurol 2019;18(9):891-902 Doi:10.1016/ s1474-4422(19)30146-2

13. Kristoffersen ES and Lundqvist C. Medicationoveruse headache: epidemiology, diagnosis and treatment. Ther Adv Drug Saf 2014;5(2):87-99 Doi: $10.1177 / 2042098614522683$

14. Sances G, Ghiotto N, Galli F, Guaschino E, Rezzani C, Guidetti $V$ and Nappi G. Risk factors in medicationoveruse headache: a 1-year follow-up study (care II protocol). Cephalalgia 2010;30(3):329-336 Doi:10.1111/j.1468-2982.2009.01934.x

15. Nagel V, Cavanagh S, Olivier M, Larripa N, Gutierrez MT, Grandinetti $M$, . . . Goicochea MT. Frequency of diagnoses in a specialized headache clinic in Buenos Aires. Headache Med 2019;10(3):66-69 Doi: 10.5935/2178-7468.20190002

16. Diener HC, Holle D, Solbach Kand Gaul C. Medicationoveruse headache: risk factors, pathophysiology and management. Nat Rev Neurol 2016; 12 (10):575-583 Doi:10.1038/nrneurol.2016.124 
17. Alstadhaug KB, Ofte HK and Kristoffersen ES Preventing and treating medication overuse headache. Pain Rep 2017;2(4):e612 Doi:10.1097/ pr9.0000000000000612

18. Find NL, Terlizzi R, Munksgaard SB, Bendtsen L, Tassorelli C, Nappi G, . . . Jensen R. Medication overuse headache in Europe and Latin America: general demographic and clinical characteristics, referral pathways and national distribution of painkillers in a descriptive, multinational, multicenter study. J Headache Pain 2015;17:20 Doi:10.1186/ s10194-016-0612-2

19. Bottiroli S, Allena M, Sances G, De Icco R, Avenali $M$, Fadic $R$, . . Tassorelli $C$. Changes in anxiety and depression symptoms associated to the outcome of MOH: A post-hoc analysis of the Comoestas Project. Cephalalgia 2018;38(4):646-654 Doi:10.1177/0333102417704415

20. Jonsson $P$, Linde $M$, Hensing $G$ and Hedenrud $T$. Sociodemographic differences in medication use, health-care contacts and sickness absence among individuals with medication-overuse headache. J Headache Pain 2012;13(4):281-290 Doi:10.1007/ s10194-012-0432-y
21. Lai JT, Dereix JD, Ganepola RP, Nightingale PG, Markey KA, Aveyard PN and Sinclair AJ. Should we educate about the risks of medication overuse headache? J Headache Pain 2014;15(1):10 Doi: 10.1186/1129-2377-15-10

22. Bekkelund SI and Salvesen R. Drug-associated headache is unrecognized in patients treated at a neurological centre. Acta Neurol Scand 2002;105(2):120-123 Doi:10.1034/j.1600-0404.2002.10193.x

23. Carlsen LN, Munksgaard SB, Jensen RH and Bendtsen L. Complete detoxification is the most effective treatment of medication-overuse headache: A randomized controlled openlabel trial. Cephalalgia 2018;38(2):225-236 Doi: $10.1177 / 0333102417737779$

24. Munksgaard SB, Madsen SK and Wienecke T. Treatment of medication overuse headache-A review. Acta Neurol Scand 2019;139(5):405-414 Doi:10.1111/ane.13074

25. Andrasik F, Grazzi L, Usai S, Kass S and Bussone $G$. Disability in chronic migraine with medication overuse: treatment effects through 5 years. Cephalalgia 2010;30(5):610-614 Doi:10.1111/ j. 1468-2982.2009.01932.x 